University of Wollongong

Research Online

Faculty of Engineering and Information

Faculty of Engineering and Information

Sciences - Papers: Part B

Sciences

2019

Flexible surface electrodes targeting biopotential signals from forearm muscles for control of prosthetic hands: Part 1 - Characterisation of semg electrodes

Siobhan O'Brien

University of Wollongong, sob832@uowmail.edu.au

Thomas Searle

University of Wollongong, tas902@uowmail.edu.au

Gursel Alici

University of Wollongong, gursel@uow.edu.au

Follow this and additional works at: https://ro.uow.edu.au/eispapers1

Part of the Engineering Commons, and the Science and Technology Studies Commons

Research Online is the open access institutional repository for the University of Wollongong. For further information contact the UOW Library: research-pubs@uow.edu.au 


\title{
Flexible surface electrodes targeting biopotential signals from forearm muscles for control of prosthetic hands: Part 1 - Characterisation of semg electrodes
}

\author{
Abstract \\ This study is Part 1 of two studies which investigate the use of various flexible surface sensors as an \\ alternative to the gold standard $\mathrm{Ag} / \mathrm{AgCl}$ surface electromyography (sEMG) electrodes in identifying \\ movement intention from a user during common hand gestures. Three conductive textiles, two \\ commercial conductive elastomers and one E-skin elastomer produced on site were tested as \\ biopotential electrodes to establish the efficacy of each in gathering movement intention from the human \\ brain at the level of the muscle. Testing was performed in vivo on two participants across three hand \\ gestures, with results demonstrating that sEMG electrodes made from a commercially sourced \\ conductive fabric can outperform the traditional $\mathrm{Ag} / \mathrm{AgCl}$ sEMG electrodes, obtaining substantially larger \\ peak and RMS measurements. Given the disadvantages of $\mathrm{Ag} / \mathrm{AgCl}$ electrodes over long usage periods, \\ namely their tendency to dry out and significant skin preparation, resulting in variable impedances and \\ skin irritation respectively, the incorporation of flexible surface EMG electrodes in hand prosthetic control \\ systems would increase functionality of the prosthetic devices, consequently increasing the quality of life \\ of prosthetic hand users.

\section{Disciplines} \\ Engineering | Science and Technology Studies

\section{Publication Details} \\ O'Brien, S., Searle, T. \& Alici, G. (2019). Flexible surface electrodes targeting biopotential signals from \\ forearm muscles for control of prosthetic hands: Part 1 - Characterisation of semg electrodes. IEEE/ \\ ASME International Conference on Advanced Intelligent Mechatronics, AIM (pp. 1019-1024). United \\ States: IEEE.
}




\title{
Flexible Surface Electrodes Targeting Biopotential Signals from Forearm Muscles for Control of Prosthetic Hands: Part 1 - Characterisation of sEMG Electrodes
}

\author{
Siobhan O'Brien, Thomas Searle and Gursel Alici, IEEE Member
}

\begin{abstract}
This study is Part 1 of two studies which investigate the use of various flexible surface sensors as an alternative to the gold standard Ag/AgCl surface electromyography (sEMG) electrodes in identifying movement intention from a user during common hand gestures. Three conductive textiles, two commercial conductive elastomers and one E-skin elastomer produced on site were tested as biopotential electrodes to establish the efficacy of each in gathering movement intention from the human brain at the level of the muscle. Testing was performed in vivo on two participants across three hand gestures, with results demonstrating that sEMG electrodes made from a commercially sourced conductive fabric can outperform the traditional Ag/AgCl sEMG electrodes, obtaining substantially larger peak and RMS measurements. Given the disadvantages of $\mathrm{Ag} / \mathrm{AgCl}$ electrodes over long usage periods, namely their tendency to dry out and significant skin preparation, resulting in variable impedances and skin irritation respectively, the incorporation of flexible surface EMG electrodes in hand prosthetic control systems would increase functionality of the prosthetic devices, consequently increasing the quality of life of prosthetic hand users.
\end{abstract}

\section{INTRODUCTION}

The need for more advanced prosthetic devices has risen in recent years, due to the increasing rate of amputations worldwide [1]. Current prosthetic devices suffer significant limitations including poor interfacing with the body, high costs, significant weights and displeasing aesthetics [2,3], resulting in high rejection rates and depression among amputees [4-5]. In order to increase the quality of life of amputees, prosthetics with greater functional capabilities and reduced costs must be designed and implemented in society.

Hand prostheses are among the most complex prosthetic devices. Knowledge from multiple disciplines including anatomy, physiology, electronics, mechanical design and software must be interwoven to simulate the abilities of a human hand [4]. Due to the complexity of the movements of the hand, EMG electrodes have been employed by many researchers to obtain biopotential signals from the forearm muscles to control hand prostheses [7-9].

Biopotentials are ionic currents which proliferate from the electrochemical activity of excitable or active cells, present in deep tissues within the nervous, muscular or endocrine systems $[10,11]$. The ionic currents, caused by a change in

This work has been supported by the funds in Intelligent Nano-Tera Systems Research Laboratory of University of Wollongong.

S. O’Brien (siobhano@uow.edu.au), T. Searle and G. Alici (corresponding author) are with the School of Mechanical, Materials, Mechatronic and Biomedical Engineering, University of Wollongong, New South Wales, 2522, Australia (e-mail: gursel@uow.edu.au). potential of an active cell membrane, travel from one cell to another through chloride ion rich body fluids [10]. Biopotential signal acquisition by skin surface electrodes involves the conversion of the body's ionic currents to electric currents by a transducer [10-13], giving readable data.

Standard electrodes used commercially for biopotential signal acquisition are made from $\mathrm{Ag} / \mathrm{AgCl}$, with an electrolytic gel employed to form and maintain a conductive interface between the electrode and the skin. These 'wet electrodes' exhibit many advantageous characteristics, including their simple, lightweight design, excellent signal to noise ratio, reliability and cost effectiveness, which make them suitable for short term biopotential measurements [1416]. In longer duration, and more complex measurements, limitations including abrasion of the skin during preparation, long application and stabilisation times, drying of electrolytic gel and resulting increase in electrode-skin impedance do arise [17-22].

In an attempt to make biopotential signal acquisition more successful in longer duration experiments, including in myoelectric prosthesis applications, several novel interfacing 'dry' electrode designs have been investigated. Dry electrodes on the whole are gaining popularity as they do not employ electrolytic gel, and thereby have higher success rates in long term use [23]. Based on the capacitive detecting principle, rather than electrode-electrolyte interface, these electrodes show excellent signal quality, though are often bulky, due to additional electronics, and are expensive [24]. Several types of dry electrodes have been examined by researchers including some incorporating conducting fibres, textiles, micro-spikes and nanostructures [25].

Electrodes and piezoresistive sensors made from conductive threads have been employed successfully in several biopotential signal acquisition applications [26,27]. Loriga, Taccini et al. [26] integrated such conductive textile electrodes into the underwear of firefighters to record an electrocardiogram (ECG), electrical activity of the heart, during emergencies. The fabric electrodes utilised were comprised of a stainless steel wire twisted around a basal yarn. This form of fabric electrode is used throughout the literature [28,29]. Another study demonstrated that smart fabric and interactive textile systems using conductive electrodes can be employed as an effective interface between neural intention and a prosthetic hand [27]. One hundred electrodes were arranged in a sleeve produced in collaboration with SMARTEX [30] which covered the upper and lower arms. Participants wearing the sleeve were asked 
to perform 9 wrist and hand movements when the arm was positioned in a neutral resting position with the forearm semi-pronated and elbow flexed 100 degrees. Results obtained illustrated that signals could be classified by a pattern recognition system with an average of $89.1 \pm 1.9 \%$ accuracy. These results are comparable to systems based on wet electrodes, with greater potential for everyday usage. Conductive textile electrodes allow high quality, continuous, remote monitoring of biopotential signals including electromyography, as well as boasting the advantages of comfort, reduced set up time and wearability [26].

Although a relatively new field of study, the creation of conductive elastomers allows for a wide variety of mechanical and electrical properties including stretchability and conductivity, meaning that products can be customised to serve a certain function [31]. A recent study proposed a flexible conductive elastomer based sensor which could be used in electromyography for signal acquisition [32]. These electrodes were superior to the gold standard $\mathrm{Ag} / \mathrm{AgCl}$ electrodes in that impedance values remained constant over time. Other studies have employed carbon nanotube/polymer hybrid technology to create effective biopotential electrodes. Results from such studies indicate little to no reduction in signal quality over time, and rigorous movement seemed to have no effect on electrode fidelity [33]. This design could easily be applied as an sEMG electrode.

In this study, six conductive materials were tested for their ability to act as dry sEMG sensors for applications such as use in a hand prosthetic control system in order to eradicate some of the disadvantages of $\mathrm{Ag} / \mathrm{AgCl}$ electrodes. A series of tests on two participants in which common hand gestures were performed were used to ascertain the most effective material. The results of each material were compared to the gold standard $\mathrm{Ag} / \mathrm{AgCl}$ electrode, most commonly used for sEMG recording. In the companion paper [34], the same materials are first characterised and subsequently employed as strain sensors to ascertain whether this modality of sensor can measure motor intention with similar efficacy. Together, this work provides a possible alternative for the sEMG systems currently used in many upper-body prosthetics.

\section{MATERIALS AND METHODS}

\section{A. Materials}

Sensors made of six materials were characterised throughout this study, five of which were inexpensive and commercially sourced, and one created on site.

1. EeonTex Conductive Stretchable Fabric by Eeonyx Corp (CA, USA). Composed of $72 \%$ nylon and $28 \%$ spandex, this black fabric is a bidirectionally stretchy purl knit, which has a thickness of approximately $0.38 \mathrm{~mm}$. The elastic filaments are coated with a long-lasting conductive formulation. The material is also extremely flexible, boasting an $85 \%$ warp recovery, and claims to be suitable for stretch applications, particularly when conformability to irregular shapes is required.

2. Knit conductive fabric luxe, P1167B, by Microwear (Chatswood, Australia). This material is $100 \%$ silver and therefore highly conductive, though has the drawback of reduced breathability, and therefore is a less comfortable fabric to wear.

3. Knit jersey conductive fabric, P1364, by Microwear (Chatswood, Australia), is comprised of $63 \%$ cotton, 35\% silver yarn and $2 \%$ spandex. The knit is composed of a single strand of yarn which traverses back and forth across the fabric.

4. ChoSeal 1215, a Ag/Cu-Silicone produced by Parker Chomerics (MA, USA) is a conductive elastomer used in industrial EMI gasket applications.

5. ChoSeal S6305, a Ni/C-Silicone produced by Parker Chomerics (MA, USA), is a similar conductive elastomer to the ChoSeal 1215.

6. 3wt \% CNT Ecoflex Conductive elastomer or E-skin. This material incorporated multi-walled carbon nanotubes (MWCNT) provided by Nanoamor (purity 95\%, outer mean diameter $=20-40 \mathrm{~nm}$; length $=5-15 \mu \mathrm{m}$ ) in an Ecoflex 0030 matrix. Once prepared, the solution was spread onto a clean glass surface, and casted with a casting knife to ensure a smooth and controlled final thickness of 300 microns [35].

\section{B. sEMG Material Testing}

Materials underwent testing on two participants to determine the ability of each to measure EMG signals. A Myoware Muscle Sensor, produced by Advanced Technologies (USA), was utilised to conduct this experiment. The device is compact, measuring approximately $50 \mathrm{~mm}$ by $20 \mathrm{~mm}$, and traditionally utilises $\mathrm{Ag} / \mathrm{AgCl}$ electrodes which clip into the circuit board via snap fasteners. During experimentation, the two signal electrodes were placed over the centre of the targeted muscle belly, in the direction of the muscle fibres of the active muscle, and the ground electrode positioned on the nearest bony landmark to provide a consistent reference signal.

The materials were cut into $15 \mathrm{~mm}$ by $15 \mathrm{~mm}$ squares, with snap fasteners sewn onto one side of all materials, evident in Fig. 1, excluding the Ecoflex Conductive Elastomer E-skin due to its delicateness. The number of stitches employed to attach the material to the press stud was minimised to ensure the material had a consistent contact with the skin. In the case of the Ecoflex Conductive Elastomer E-Skin, the sheet of material was placed up against the snap fastener of the Myoware sensor to establish a connection. A thin sheet of cardboard with circular holes cut from it was placed between the Myoware Muscle Sensor and the electrodes to prevent contact between conductive materials and the circuit. Prior to electrode placement, the skin was prepared by shaving,

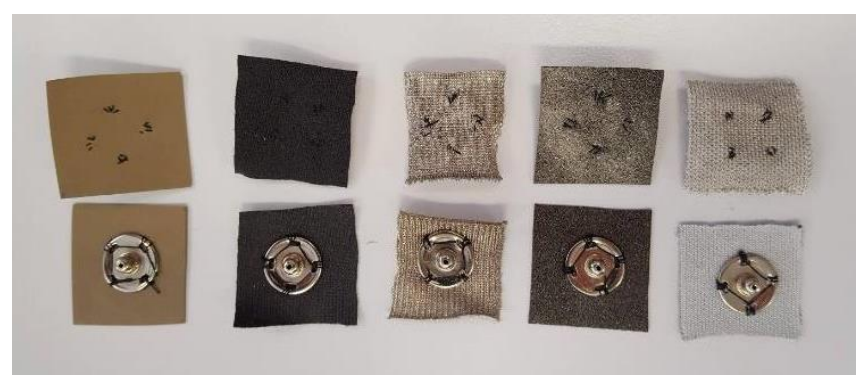

Fig. 1: Snap fasteners sewn onto material electrodes 
abrading and wiping the abraded area with rubbing alcohol to clean the skin and remove the uppermost layer of dead skin cells. This preparation is particularly important for the $\mathrm{Ag} / \mathrm{AgCl}$ electrodes to reduce electrode-skin impedance.

Three common hand gestures, shown in Fig. 2, were undertaken with each of the experimental materials as 'dry' sEMG electrodes. The results of tests recorded with these materials were compared to the results of $\mathrm{Ag} / \mathrm{AgCl}$ electrode, the gold standard of sEMG electrodes. In all experiments, the ground electrode was an $\mathrm{Ag} / \mathrm{AgCl}$ electrode, in order to keep the reference signal consistent amongst materials. The three tested gestures were 'Hand Open', 'Closed Fist' and 'Index Finger Point'. Table 1 documents the placement of the electrodes for best recording of muscle signals in each gesture. Along with the electrode placement documentation, muscles were palpated in both participants prior to testing, and locations for electrode placement marked with permanent markers for consistency between materials. The Myoware Muscle Sensor was held in place on the forearm using a bandage during all testing.

The data across three trials for each material in each gesture was averaged, smoothed and plotted with a standard error envelope showing variation between individual trials. The RMS value of the muscle activation was calculated, along with the average peak activation across three tests for each material on each participant, to allow for comparisons to be made between materials.

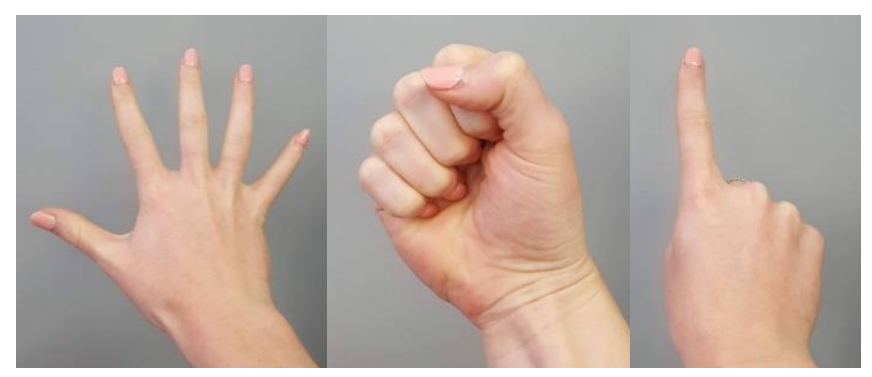

Fig. 2: Gestures tested for sEMG experiments

Table 1: Electrode placement for hand gestures

\begin{tabular}{|l|l|}
\hline Gesture & Electrode Placement \\
\hline $\begin{array}{l}\text { Hand } \\
\text { Open }\end{array}$ & $\begin{array}{l}\text { Posterior middle forearm, one third of the } \\
\text { distance from the olecranon process to the } \\
\text { styloid process of the ulna over palpable } \\
\text { muscle mass of Extensor Digitorum (ED) }\end{array}$ \\
\hline $\begin{array}{l}\text { Closed } \\
\text { Fist }\end{array}$ & $\begin{array}{l}\text { Ventral-medial forearm, one fifth of the } \\
\text { distance between the medial epicondyle of the } \\
\text { humerus and the pisiform bone, over the } \\
\text { muscle belly of Flexor Digitorum Superficialis }\end{array}$ \\
\hline $\begin{array}{l}\text { Index } \\
\text { Finger } \\
\text { Point }\end{array}$ & $\begin{array}{l}\text { Posterior lateral forearm, midway between the } \\
\text { olecranon process and the styloid process of } \\
\text { the ulna over palpable muscle mass of Extensor } \\
\text { Indicis (EI) }\end{array}$ \\
\hline
\end{tabular}

\section{RESULTS AND DISCUSSIONS}

Two participants underwent testing in which the three aforementioned gestures were performed: Hand Open, Closed Fist and Index Finger Point, with three trials recorded for each material. Results for each material during the 'Hand Open' gesture performed by Participant 2 are portrayed the plots shown in Fig. 3. The peak in each of the graphs corresponds to the point of greatest muscle activation recorded by the Myoware Sensor, at the end point of the movement in question.

The two silver knitted materials, the Luxe Silver Knit and the Jersey Knit, along with the $\mathrm{Ni} / \mathrm{C}$-Silicone performed poorly throughout all gestures across both participants. As evident in Fig. 3a, the Ni/C-Silicone electrodes were unable to consistently produce a response greater than the baseline reading of approximately $40 \mathrm{mV}$, whilst the Jersey Knit fabric also failed to detect any response from the gesture, performed 1 second into the trial. The poor functionality displayed by the Jersey Knit fabric is hypothesised to be a result of the nature of the knit. When a digital multi-meter is used to measure the static resistance of the material, only the darker side, as evident in Fig. 1, is conductive, as this is the side on which the silver yarn loops are visible. When placed on the skin, the conductive side of the material must be placed downwards to collect biopotential data from the muscle, though this means the snap fastener, when sewn to the lighter side of the material is mostly insulated from the conductive fibres. A slightly greater degree of success is achieved by the Luxe Silver Knit, shown in Fig. 3c, which has peaks which reflect that of the commercial standard in Fig. 4. However this material has greater inconsistencies in baseline and peak readings, and displays secondary peaks post contraction in this 'Hand Open' gesture. The Luxe Silver Knit also performed erratically in other gestures.

As evident in Fig. 3d, the 3wt\% CNT E-skin performed inconsistently in the 'Hand Open' trial, unable to detect any activity. Comparatively, during all three trials for the 'Hand Open' gesture performed by Participant 1, the signal 'maxes out' when the contraction occurs. This is due to the saturation of the ADC in the Myoware sensor. The gain of the system could be manually altered to avoid this phenomenon. Assuming the electrodes were well positioned and did not lose contact throughout contraction, this result suggests the E-skin has a promising capacity to operate as an EMG electrode. In the remaining trials, no reliable muscle activation was detected by the E-skin. The baseline reading of approximately $40 \mathrm{mV}$ in most tests is consistent with other unsuccessful materials, and is equal to the reading obtained from the reference electrode.

Across the three gestures, only Eeontex Conductive Fabric and the $\mathrm{Ag} / \mathrm{Cu}-\mathrm{Silicone}$, along with the gold standard $\mathrm{Ag} / \mathrm{AgCl}$ electrodes consistently detect EMG data from underlying muscles. Tables 2 and 3 outline the mean peak muscle activation, and mean RMS value for the window of activation in each of the gestures for these three materials. The EMG data obtained by the $\mathrm{Ag} / \mathrm{Cu}$-Silicone across all gestures is highly consistent, and very closely reflects that of the commercial $\mathrm{Ag} / \mathrm{AgCl}$ electrode, as evident in Fig 3e, the 

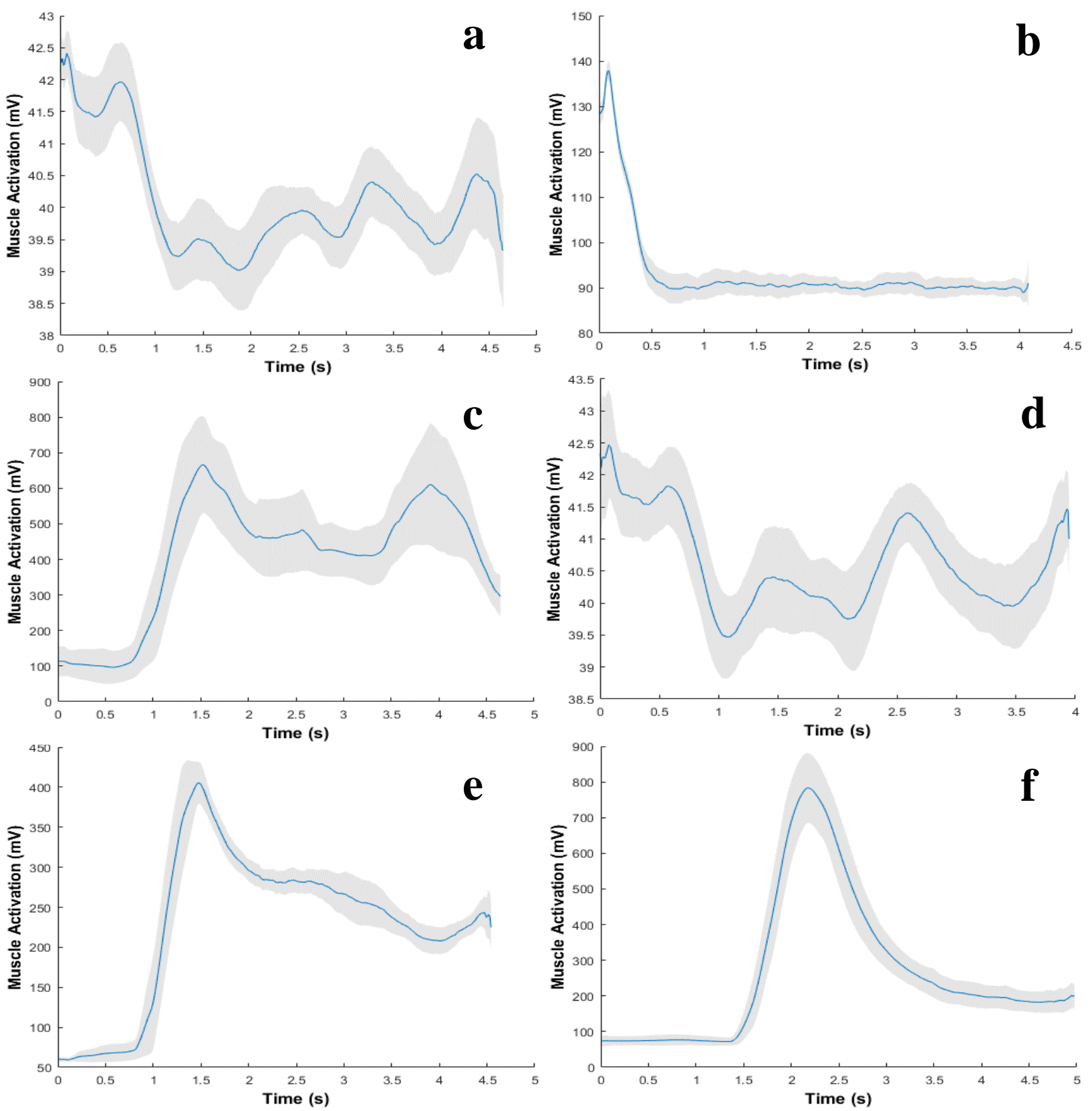

Fig. 3: EMG Signals for Hand Open by Participant 2 a) Ni/C Silicone b) Jersey Knit c) Luxe Silver Knit d) 3wt\% CNT E-skin e) Ag/Cu Silicone f) Eeontex

'Hand Open' testing results for Participant 2. From Tables 2 and 3 , the material displayed superiority in signal acquisition when compared to $\mathrm{Ag} / \mathrm{AgCl}$ in Participant 1, though remained on par with the gold standard for Participant 2. In the 'Hand Open' Gesture shown, both the Ag/Cu-Silicone and Eeontex fabric observe peak values within $12 \%$ of that of the $\mathrm{Ag} / \mathrm{AgCl}$ electrode. A significant result evident in the 'Closed Fist' gesture test across both subjects, and the 'Hand Open' gesture in Fig. 3f, is the substantially larger peak and RMS values observed by the Eeontex fabric. The fabric demonstrates high stability and consistency across all trials, with peak values of $776 \mathrm{mV}$ and $840 \mathrm{mV}$ for Participants 1 and 2, respectively. This value is approximately 4 times that recorded by the $\mathrm{Ag} / \mathrm{AgCl}$ material suggesting that the Eeontex fabric is more suited to EMG electrode applications, as the quality and amplitude of the signal is greater than the gold standard commercial electrode.

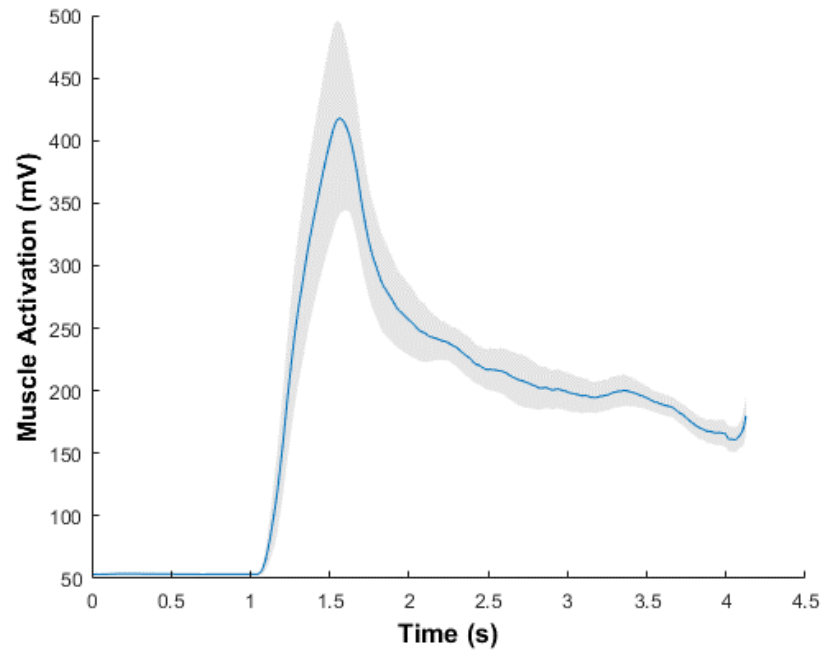

Fig. 4: EMG Signals for $\mathrm{Ag} / \mathrm{AgCl}$ Electrode during Hand Open Gesture 
Table 2: Summary of sEMG testing results Participant 1

\begin{tabular}{|l|l|l|l|l|l|l|}
\cline { 2 - 7 } \multicolumn{1}{c|}{} & \multicolumn{2}{l|}{$\begin{array}{l}\text { Hand Open } \\
\text { (mV) }\end{array}$} & \multicolumn{2}{l|}{$\begin{array}{l}\text { Closed Fist } \\
(\mathbf{m V})\end{array}$} & \multicolumn{2}{l|}{$\begin{array}{l}\text { Index Finger } \\
\text { Point (mV) }\end{array}$} \\
\hline Material & Peak & RMS & Peak & RMS & Peak & RMS \\
\hline Ag/AgCl & 444.2 & 255.0 & 213.3 & 124.5 & 158.4 & 119.1 \\
\hline Eeontex & 394.7 & 268.1 & 776.0 & 365.6 & 358.5 & 238.9 \\
\hline $\begin{array}{l}\text { Ag/Cu- } \\
\text { Silicone }\end{array}$ & 411.7 & 219.1 & 701.0 & 303.8 & 269.6 & 197.7 \\
\hline
\end{tabular}

Table 3: Summary of sEMG testing results Participant 2

\begin{tabular}{|l|l|l|l|l|l|l|}
\cline { 2 - 7 } \multicolumn{1}{c|}{} & \multicolumn{2}{l|}{$\begin{array}{l}\text { Hand Open } \\
\text { (mV) }\end{array}$} & \multicolumn{2}{l|}{$\begin{array}{l}\text { Closed Fist } \\
\text { (mV) }\end{array}$} & \multicolumn{2}{l|}{$\begin{array}{l}\text { Index Finger } \\
\text { Point (mV) }\end{array}$} \\
\hline Material & Peak & RMS & Peak & RMS & Peak & RMS \\
\hline Ag/AgCl & 460.5 & 260.2 & 222.1 & 125.7 & 313.3 & $226 / 3$ \\
\hline Eeontex & 821.4 & 391.4 & 840.5 & 383.4 & 541.3 & 299.2 \\
\hline $\begin{array}{l}\text { Ag/Cu- } \\
\text { Silicone }\end{array}$ & 420.4 & 273.7 & 198.9 & 98.1 & 478.7 & 269.1 \\
\hline
\end{tabular}

It is difficult to compare consistency among participants and between trials of differing materials, as the force produced by targeted muscles was not monitored. Although the instruction of a maximal voluntary contraction (MVC) was given to the participant prior to testing, the maximal force produced may reduce over a number of trials with an increase in muscular fatigue. Variation between participants may also have been a result of electrode placement. Placing a sensor too far from the midline or main muscle belly will reduce signal strength and clarity, as fewer motor units are measured, and interference due to cross talk arises [36].

For use in a prosthetic control system, the variety of gestures being performed must be considered before making a decision on which material to use as a dry electrode. The location, depth and size of the targeted muscles will influence the signal quality obtained due to variations in skin thickness, and cross talk. The skin over Flexor Digitorum Superficialis on the anterior forearm, the targeted muscle for 'Closed Fist,' is considerably thinner than that of the posterior forearm, and the muscle mass dedicated to flexion of the fingers is larger than that for finger extension [37], explaining the consistency seen in the results for this gesture. Comparatively, in performing the pointing gesture, cross talk will arise in the system, as ED co-activates with EI to extend the index finger [37]. Cross talk occurs when neighbouring muscles contribute significantly to the captured EMG signal. Typically cross talk does not exceed $10 \%-15 \%$ of the overall signal contents, though may be more significant when attempting to receive a signal from a deeper or smaller muscle [37]. Considering that other gestures often performed by prosthetic hands, including 'Thumbs Up' and 'Pinching', require the acquisition of biopotential signals from other smaller muscles, only material samples which were consistent, stable and large enough to detect differences in muscle activation should be considered for sEMG electrodes.

From the results presented, it is concluded that the material which has the greatest capacity to function as an
EMG electrode to obtain biopotential signals such as muscle potentials is the Eeontex Fabric. This fabric shows consistently higher peak and RMS values for the maximum voluntary muscle contraction undertaken by both participants when compared to the gold standard, and is stable over the three gestures. This result may be due to the nature of the Eeontex Conductive Fabric, as the yarn is coated in a conductive formulation. The conductive component of this material is therefore directly touching the skin during EMG testing, and is able to effectively record muscle activation data as a result. Comparatively, the Jersey Knit has only a component of conductive yarn, reducing the conductive contact area, and the conductive component of the elastomer materials is dispersed within a matrix, also reducing direct contact between skin and conductive substance. This is a significant finding, as a myoelectric prosthetic device with conductive textile electrodes would provide greater comfort for the user, and can be more functional for everyday use. Ultimately, this reflects the sentiment of Farina, Lorrain et al. [28], that conductive textiles are very much a viable alternative to 'wet' sEMG electrodes.

\section{CONCLUSION}

Although significant advancements have been made in research on myoelectric prostheses in recent decades, this has not been reflected in a reduction in rejection rates of the devices [8]. The aim of this study is to investigate muscle activation via EMG using unconventional electrode materials as a possible prosthetic control system for the identification of user intention for a prosthetic hand device. From the analysis of results from gesture experiments undertaken on two participants, it is concluded that the material which has the greatest potential to act as an sEMG electrode in a hand prosthetic control application is the Eeontex conductive textile. Eeontex is highly sensitive, consistent in measurements, and demonstrates consistently higher peak and RMS values for the maximum voluntary muscle contraction when compared to $\mathrm{Ag} / \mathrm{AgCl}$ electrodes. This is advantageous for a prosthetic control system, as the change in amplitude with movement is more easily discernible. This bidirectionally stretchy, isotropic fabric is able to be washed, with tests undertaken by the manufacturer proving that no appreciable increase in resistivity is observed after 30 wash cycles. The material is comfortable and breathable, boasting a high conformability to irregular shapes, an advantageous characteristic for the intended application, with good durability and high biocompatibility. Materials including conductive textiles and elastomers show great potential to eventually improve general acceptance of prosthetic devices, reduce rejection rates and increase the quality of life attained by prosthetic hand users. However, further research is required before such 'dry' materials can replace the gold standard $\mathrm{Ag} / \mathrm{AgCl}$ sEMG electrode currently used in the control of myoelectric prostheses. The companion paper further builds on the work presented in this study, questioning whether the same materials could effectively measure user intention by acting as biometric strain sensors [34]. 


\section{ACKNOWLEDGMENT}

This research is supported by the research funds available in Intelligent Nano-Tera Systems Research Laboratory of University of Wollongong.

\section{REFERENCES}

[1] Ziegler-Graham, Kathryn, et al. (2008) "Estimating the prevalence of limb loss in the United States: 2005 to 2050." Archives of physical medicine and rehabilitation 89.3: 422-429.

[2] Atkins, D. J., D. C. Y. Heard and W. H. Donovan (1996). "Epidemiologic overview of individuals with upper-limb loss and their reported research priorities." Journal of Prosthetics \& Orthotics (JPO) 8(1): 2-11.

[3] Geethanjali, P. (2016). "Myoelectric control of prosthetic hands: stateof-the-art review." Medical Devices: Evidence and Research, Vol 2016, Iss Issue 1, Pp 247-255 (2016)(Issue 1): 247.

[4] Bollinger, C. (2016). "Moving Forward After Upper Limb Loss." Rehab Management: The Interdisciplinary Journal of Rehabilitation: 36-40.

[5] Biddiss, E. and T. Chau (2007). "The roles of predisposing characteristics, established need, and enabling resources on upper extremity prosthesis use and abandonment." Disability \& Rehabilitation: Assistive Technology 2(2): 71-84.

[6] Singh, R., D. Ripley, B. Pentland, I. Todd, J. Hunter, L. Hutton and A. Philip (2009). "Depression and anxiety symptoms after lower limb amputation: the rise and fall." Clinical Rehabilitation 23(3): 281-286.

[7] Farina, D., T. Lorrain, F. Negro and N. Jiang (2010). "High-density EMG E-textile systems for the control of active prostheses." Conference Proceedings: ... Annual International Conference Of The IEEE Engineering In Medicine And Biology Society. IEEE Engineering In Medicine And Biology Society. Annual Conference 2010: 3591-3593.

[8] Aishwarya, R., M. Prabhu, G. Sumithra and M. Anusiya (2013). "Feature extraction for emg based prostheses control." ICTACT Journal on Soft Computing, Vol 3, Iss 2, Pp 472-477 (2013)(2): 472.

[9] Suberbiola, A., E. Zulueta, J. M. Lopez-Guede, I. Etxeberria-Agiriano and M. Graña (2015). "Arm Orthosis/Prosthesis Movement Control Based on Surface EMG Signal Extraction." International Journal Of Neural Systems 25(3): 1550009-1550009.

[10] Griss, P., P. Enoksson, H. K. Tolvanen-Laakso, P. Merilainen, S. Ollmar and G. Stemme (2001). "Micromachined electrodes for biopotential measurements." Journal of Microelectromechanical Systems 10(1): 10-16.

[11] Xie, L., G. Yang, L. Xu, F. Seoane, Q. Chen and L. Zheng (2013). "Characterization of dry biopotential electrodes." Engineering in Medicine and Biology Society (EMBC), 2013 35th Annual International Conference of the IEEE, IEEE.

[12] Kaitainen, S., A. Kutvonen, M. Suvanto, T. T. Pakkanen, R. Lappalainen and S. Myllymaa (2014). "Liquid silicone rubber (LSR)based dry bioelectrodes: The effect of surface micropillar structuring and silver coating on contact impedance." Sensors and Actuators A: Physical 206: 22-29.

[13] Forvi, E., M. Bedoni, R. Carabalona, M. Soncini, P. Mazzoleni, F. Rizzo, C. O'Mahony, C. Morasso, D. G. Cassarà and F. Gramatica (2012). "Preliminary technological assessment of microneedles-based dry electrodes for biopotential monitoring in clinical examinations." Sensors and Actuators A: Physical 180: 177-186.

[14] Baek, H. J., H. J. Lee, Y. G. Lim and K. S. Park (2012). "Conductive polymer foam surface improves the performance of a capacitive EEG electrode." IEEE Transactions on Biomedical Engineering 59(12): 3422-3431.

[15] Mota, A. R., L. Duarte, D. Rodrigues, A. Martins, A. Machado, F. Vaz, P. Fiedler, J. Haueisen, J. Nóbrega and C. Fonseca (2013). "Development of a quasi-dry electrode for EEG recording." Sensors and Actuators A: Physical 199: 310-317.

[16] Conor, O. M., G. Konstantin, C. Antonio, G. Giuseppe, K. Anan and G. Paul (2016). "Design, fabrication and skin-electrode contact analysis of polymer microneedle-based ECG electrodes." Journal of Micromechanics \& Microengineering 26(8): 1-1.
[17] Taheri, B. A., R. T. Knight and R. L. Smith (1994). "A dry electrode for EEG recording." Electroencephalography and clinical neurophysiology 90(5): 376-383.

[18] Teplan, M. (2002). "Fundamentals of EEG measurement." Measurement science review 2(2): 1-11.

[19] Baek, J.-Y., J.-H. An, J.-M. Choi, K.-S. Park and S.-H. Lee (2008), "Flexible polymeric dry electrodes for the long-term monitoring of ECG." Sensors \& Actuators: A. Physical 143: 423-429.

[20] Jung, H.-C., J.-H. Moon, D.-H. Baek, J.-H. Lee, Y.-Y. Choi, J.-S Hong and S.-H. Lee (2012). "CNT/PDMS composite flexible dry electrodesfor long-term ECG monitoring." IEEE Transactions on Biomedical Engineering 59(5): 1472-1479.

[21] Fiedler, P., P. Pedrosa, S. Griebel, C. Fonseca, F. Vaz, E. Supriyanto, F. Zanow and J. Haueisen (2015). "Novel Multipin Electrode Cap System for Dry Electroencephalography." Brain Topography 28(5): 647-656

[22] Paul, G., R. Torah, S. Beeby and J. Tudor (2015). "Novel active electrodes for ECG monitoring on woven textiles fabricated by screen and stencil printing." Sensors \& Actuators: A. Physical 221: 60-66.

[23] Srivastava, A. K., B. Bhartia, K. Mukhopadhyay and A. Sharma (2015). "Long term biopotential recording by body conformable photolithography fabricated low cost polymeric microneedle arrays." Sensors and Actuators A: Physical 236: 164-172.

[24] O'Mahony, C., K. Grygoryev, A. Ciarlone, G. Giannoni, A. Kenthao and P. Galvin (2016). "Design, fabrication and skin-electrode contact analysis of polymer microneedle-based ECG electrodes." Journal of Micromechanics and Microengineering 26(8): 084005.

[25] Arai, M., Y. Nishinaka and N. Miki (2015). "Electroencephalogram measurement using polymer-based dry microneedle electrode." Japanese Journal of Applied Physics 54(6S1): 06FP14.

[26] Loriga, G., N. Taccini, M. Pacelli and R. Paradiso (2007). "Flat Knitted Sensors for Respiration Monitoring [From mind to market]." IEEE Industrial Electronics Magazine 1(3): 4.

[27] Farina, D., T. Lorrain, F. Negro and N. Jiang (2010). "High-density EMG E-textile systems for the control of active prostheses." Conference Proceedings: ... Annual International Conference Of The IEEE Engineering In Medicine And Biology Society. IEEE Engineering In Medicine And Biology Society. Annual Conference 2010: 3591-3593.

[28] Paradiso, R. (2004). Knitted textile for the monitoring of vital signals, Google Patents.

[29] Paradiso, R., G. Loriga and N. Taccini (2005). "A wearable health care system based on knitted integrated sensors." IEEE transactions on Information Technology in biomedicine 9(3): 337-344.

[30] Pacelli, M., G. Loriga and R. Paradiso (2007). Flat knitted sensors for respiration monitoring. Industrial Electronics, 2007. ISIE 2007. IEEE International Symposium on, IEEE.

[31] Noh, J.S. (2016). "Conductive elastomers for stretchable electronics, sensors and energy harvesters." Polymers 8(4): 123.

[32] Das, P. S. and J.-Y. Park (2017). "A flexible touch sensor based on conductive elastomer for biopotential monitoring applications." Biomedical Signal Processing \& Control 33: 72.

[33] Peng, H.-L., J.-Q. Liu, H.-C. Tian, B. Xu, Y.-Z. Dong, B. Yang, X. Chen and C.-S. Yang (2015). "Flexible dry electrode based on carbon nanotube/polymer hybrid micropillars for biopotential recording." Sensors \& Actuators: A. Physical 235: 48-56.

[34] O'Brien, S., T. Searle, and G. Alici, "Flexible Surface Electrodes Targeting Biopotential Signals from Forearm Muscles for Control of Prosthetic Hands: Part 2 - Characterization of Substrates for Strain Sensors", 2019 IEEE International Conference on Advanced Intelligent Mechatronics, Hong Kong, July 2019.

[35] Mai, Huy, Rahim Mutlu, Charbel Tawk, Gursel Alici, and Vitor Sencadas (2019). "Ultra-stretchable MWCNT-Ecoflex piezoresistive sensors for human motion detection applications." Composites Science and Technology

[36] Serup, J., G. B. Jemec and G. L. Grove (2006). Handbook of noninvasive methods and the skin, CRC press.

[37] Konrad, P. (2005). "The abc of emg." A practical introduction to kinesiological electromyography 1: 30-35. 